\title{
Maturation and Conversion of Somatic Embryos of Three Genetically Diverse Rose Cultivars
}

\author{
Javier Castillón and Kathryn Kamo ${ }^{1}$ \\ U.S. Department of Agriculture-Agricultural Research Service, U.S. \\ National Arboretum, Floral and Nursery Plants Research Unit, Beltsville, \\ MD 20705
}

Additional index words. carbohydrate, embryogenic callus, osmoticum, regeneration, Rosa hybrida

Abstract. Embryogenic callus cultures of three genetically diverse cultivars of rose (Rosa hybrida L.), the floribunda 'Trumpeter', the multiflora 'Dr. Huey', and the hybrid tea 'Tineké', were used to study the effect of various carbohydrates and osmotically active compounds on somatic embryo maturation and conversion. Cotyledonary-stage embryos were produced by dispersing callus in liquid medium followed by filtration to isolate globular-stage embryos. Quantitative experiments were conducted to determine maturation and conversion of the three rose cultivars in response to medium with sucrose, glucose, fructose, or maltose as the primary carbon source and also in response to various concentrations of either myo-inositol, polyethylene glycol, or mannitol in combination with $3 \%$ sucrose. Conversion of $27 \%$ was achieved for 'Trumpeter' embryos following their maturation on 3\% fructose. 'Dr. Huey' embryos required maturation on medium containing $3 \%$ sucrose supplemented with either $2.5 \%$ or $5 \%$ mannitol for 36\% and $61 \%$ conversion, respectively. Maturation of 'Tineké' embryos on either $3 \%$ sucrose, $3 \%$ glucose, or $3 \%$ fructose resulted in a maximum $12 \%$ conversion.

Roses are one of the most important floral crops in the world. However, most of the modern cultivars are susceptible to various microbial and insect pests. This necessitates the regular use of fungicides and insecticides by rose growers and substantially adds to their production expenses. Molecular biology techniques are now available for genetic modification of rose in order to obtain better resistance to pathogens. While several researchers report successful transformation of rose plants (Derks et al., 1995; Firoozabady et al., 1994; Marchant et al., 1998; Souq et al., 1996; van der Salm et al., 1997), there are no commercial introductions of genetically modified cultivars with increased pathogen resistance. Part of the difficulty associated with transformation of rose is developing an efficient regeneration system, applicable to many rose cultivars, that allows many plants to be recovered.

Many rose cultivars produce embryogenic callus bearing somatic embryos for which regeneration rates vary widely among cultivars. In most cases, regeneration is too sporadic for development of efficient transformation protocols.

Several researchers report regeneration of rose plants from callus (de Wit et al., 1990; Hsia and Korban, 1996; Kintzios et al., 1999; Marchant et al., 1996; Matthews et al., 1991;

Received for publication 27 Nov. 2000. Accepted for publication $11 \mathrm{Dec}$. 2001. Use of trade names in this publication does not imply endorsement of the U.S. Dept. of Agriculture.

${ }^{1}$ To whom reprint requests should be addressed. E-mail address: kkamo@asrr.arsusda.gov
Murali et al., 1996; Noriega and Sondahl, 1991; Valles and Boxus, 1987; van der Salm et al., 1996). The methods are specific to only one or two cultivars. These previous papers are typically reports of regeneration rather than analysis of specific factors that contribute to the regeneration process, and only three of these studies report quantitative data on embryo maturation and conversion. Murali et al. (1996) show that abscisic acid and phloroglucinol stimulate conversion of somatic embryos of cv. Arizona. Marchant et al. (1996) demonstrate that somatic embryo conversion of two floribunda cultivars is enhanced by both $\mathrm{N}^{6}$-benzylaminopurine (BA) and a $4{ }^{\circ} \mathrm{C}$ cold treatment. The combination of BA with methyl laurate stimulates conversion of a hybrid cultivar (Sarasan et al., 2001). Data derived from quantitative experiments involving many somatic embryos of rose are required for further elucidation of specific factors important to somatic embryo development.

Studies of somatic embryos of Rosa rugosa Thunb. (Kunitake et al., 1993) as well as other genera (Biahoua and Bonneau, 1999; Klimaszewska et al., 1997; Schuller and Reuther, 1993; Tremblay and Tremblay, 1991) report the use of different carbohydrates as carbon sources and osmoticum to enhance maturation and conversion rate.

We present the results of quantitative studies conducted to test the effect of various carbohydrates and osmotically active compounds on maturation and conversion rate of somatic embryos from three rose cultivars. Embryogenic callus cultures used for these experiments were from the floribunda 'Trum- peter', the hybrid tea 'Tineké', and the rootstock 'Dr. Huey'. The carbohydrates used as carbon sources in the media were sucrose, glucose, fructose, and maltose. The compounds myo-inositol, PEG (polyethylene gly$\mathrm{col}$ ), and mannitol were used at various concentrations to increase the osmotic potential of the medium. Mannitol and PEG were used to compare the effects of a metabolizable osmoticum with one that is nonpermeating and non-plasmolyzing. Myo-inositol is reported to be the best osmoticum (Marchant et al., 1998) during biolistic bombardment of rose and was therefore also selected as an osmoticum in this study.

\section{Materials and Methods}

Establishment and maintenance of embryogenic cultures. Embryogenic callus cultures were established using the procedure of Marchant et al. (1996) for 'Trumpeter' and the procedure of Derks et al. (1995) for 'Tineké' and 'Dr. Huey'. Cultures of 'Tineké' were maintained on MS medium (Murashige and Skoog, 1962) with 3\% (w/v) sucrose, $18.1 \mu \mathrm{M}$ dicamba (3,6-dichloro-o-anisic acid), $0.46 \mu \mathrm{M}$ kinetin, $0.25 \%$ (w/v) Phytagel (Sigma Chemical Co., St Louis), and the following in $\mathrm{mg} \cdot \mathrm{L}^{-1}$ : glycine, 2.0; thiamine, 1.0; pyridoxine, 0.5; nicotinic acid, 0.5; myo-inositol, 100.0. Cultures of 'Trumpeter' and 'Dr. Huey' were maintained on $\mathrm{SH}$ medium (Schenk and Hildebrandt, 1972) with $3 \%(\mathrm{w} / \mathrm{v})$ sucrose, $13.6 \mu_{\mathrm{M}}$ 2,4-D (2,4-dichlorophenoxyacetic acid), $0.25 \%(\mathrm{w} / \mathrm{v}$ ) Phytagel, and the following in $\mathrm{mg} \cdot \mathrm{L}^{-1}$ : thiamine, 1.0; pyridoxine, 0.5 ; nicotinic acid, 0.5; L-proline, 300 . Both media were adjusted to $\mathrm{pH} 5.8$ prior to autoclaving 20 min at $18 \mathrm{psi}, 121{ }^{\circ} \mathrm{C}$, and then $25 \mathrm{~mL}$ of medium were placed in each $100 \times 20 \mathrm{~mm}$ Petri plate. All cultures were maintained in the dark at $25^{\circ} \mathrm{C}$ and subcultured onto fresh medium every 5-6 weeks.

Preparation of inoculum for use in embryo maturation experiments. About $20 \mathrm{~g}$ of embryogenic callus were placed in a baffled flask with $50 \mathrm{~mL}$ of medium and placed on an orbital shaker set at $124 \mathrm{rpm}$ for $2-3 \mathrm{~h}$ to break up the callus. Medium used for 'Trumpeter' and 'Dr. Huey' consisted of SH salts and vitamins and $1.5 \%$ sucrose. Medium used for 'Tineké' consisted of MS salts and vitamins, $100 \mathrm{mg} \cdot \mathrm{L}^{-1}$ myo-inositol, $2.0 \mathrm{mg} \cdot \mathrm{L}^{-1}$ glycine, and $1.5 \%$ sucrose. The suspension produced by shaking was sequentially filtered through stainless steel sieves with mesh sizes of 2000,1180 , and $850 \mu \mathrm{m}$. This eliminated cotyledonary-stage embryos and provided a mixture of globular-stage embryos, proembryogenic masses, and nonembryogenic callus cells. Additional medium was used during this process as needed to wash cells through the sieves. Cells that passed through the $850-\mu \mathrm{m}$ mesh were washed three times with the same medium used for filtering, but without sucrose, and resuspended in 150$200 \mathrm{~mL}$ of this medium. Plates $(100 \times 20 \mathrm{~mm})$ were inoculated using $2 \mathrm{~mL}$ of the suspension (three plates per treatment) and then placed in the dark at $25^{\circ} \mathrm{C}$. 
Maturation medium. The base medium for testing various carbohydrates and osmotica consisted of MS salts and vitamins, $100 \mathrm{mg} \cdot \mathrm{L}^{-1}$ myo-inositol, $2 \mathrm{mg} \cdot \mathrm{L}^{-1}$ glycine, $0.25 \%(\mathrm{w} / \mathrm{v})$ Phytagel, $0.5 \%(\mathrm{w} / \mathrm{v})$ activated charcoal (AC), and $500 \mathrm{mg} \cdot \mathrm{L}^{-1}$ MES (2-[nmorpholino]ethanesulfonic acid). The carbohydrates tested included $3 \%(\mathrm{w} / \mathrm{v})$ of either sucrose, glucose, fructose, or maltose. Media were prepared without either charcoal or Phytagel in $60 \%$ of the medium's final volume and filter-sterilized to avoid degradation of the carbohydrates during autoclaving. The medium was adjusted to $\mathrm{pH} 5.8$ prior to filtersterilization through $0.2-\mu \mathrm{m}$ filters. A solution containing AC and Phytagel in $40 \%$ of the final volume was autoclaved and added to filter-sterilized medium to give the final volume and concentration of all components. Cotyledonary-stage embryos in each plate were counted after 4-5 weeks on maturation medium. Only embryos with expanded cotyledons that were $2 \mathrm{~mm}$ or greater in diameter were scored as mature.

The osmotically active compounds were either $2.5 \%, 5.0 \%$, or $7.5 \%(\mathrm{w} / \mathrm{v})$ myoinositol, PEG (m.w. 3350) or mannitol, and each was added to the base medium containing $3 \%(\mathrm{w} / \mathrm{v})$ sucrose. Base medium containing $3 \%$ sucrose was used as a $0 \%$ osmoticum control. A solution containing the Phytagel,
AC, and osmoticum was autoclaved in $94 \%$ of the total volume, cooled to $60^{\circ} \mathrm{C}$, and the remaining components added using sterile 50X stock solutions (Murashige and Skoog Complete Medium, 50× Concentrate; Gibco BRL/Life Technologies, Grand Island, N.Y.). The osmotic potential of each medium was measured in three replications using a 5500 Wescor Vapor Pressure Osmometer (Wescor, Logan, Utah).

Conversion of cotyledonary-stage embryos. Cotyledonary-stage embryos were cultured 10-11 weeks, without subculture, on each maturation medium containing various carbohydrates and osmoticum and then transferred to conversion medium (MS salts and vitamins, $3 \%$ sucrose, $100 \mathrm{mg} \cdot \mathrm{L}^{-1}$ myoinositol, $2 \mathrm{mg} \cdot \mathrm{L}^{-1}$ glycine, $0.5 \% \mathrm{AC}, 0.25 \%$ Phytagel). Cotyledonary-stage embryos, 150 200 taken from three plates, were transferred to conversion medium and kept in the dark for 1 week and then placed under cool-white fluorescent lights $\left(10-20 \mu \mathrm{mol} \cdot \mathrm{m}^{-2} \cdot \mathrm{s}^{-1}\right)$ with a 12-h photoperiod. The percentage of embryos that converted was determined by counting the number of embryos with both shoots and roots after 6 weeks on conversion medium.

Statistical analysis. An analysis of variance using Duncan's new multiple range test with a $95 \%$ confidence interval $(P<0.05)$ was used for comparing the means of the osmotic and carbohydrate treatments for each cultivar (Figs. 2-5). A total of 150-200 embryos was isolated by filtration, and either three or four plates of 50 embryos each were prepared for each treatment. Each plate of 50 embryos was treated as a replicate.

\section{Results and Discussion}

Maturation of somatic embryos on various carbon sources. Mature, cotyledonary-stage embryos of the three cultivars, 'Trumpeter', 'Dr. Huey', and 'Tineké', developed following isolation of globular-stage embryos from embryogenic callus (Fig. 1 A and B). There were two obvious similarities in the way the cultivars responded to the four carbohydrates tested. First, no other carbohydrate induced more embryo maturation than sucrose (Fig. 2). These results were in contrast to those obtained with Rosa rugosa embryogenic cultures in which medium with glucose, fructose, or maltose all produced more somatic embryos than sucrose (Kunitake et al., 1993). Second, $3 \%$ maltose produced the fewest embryos in all three cultivars (Fig. 2). Marchant et al. (1996) used maltose in medium for germination of somatic embryos of 'Trumpeter' following their maturation on a medium containing sucrose. In loblolly pine, $6 \%$ maltose enhanced embryo maturation $\approx 10$-fold
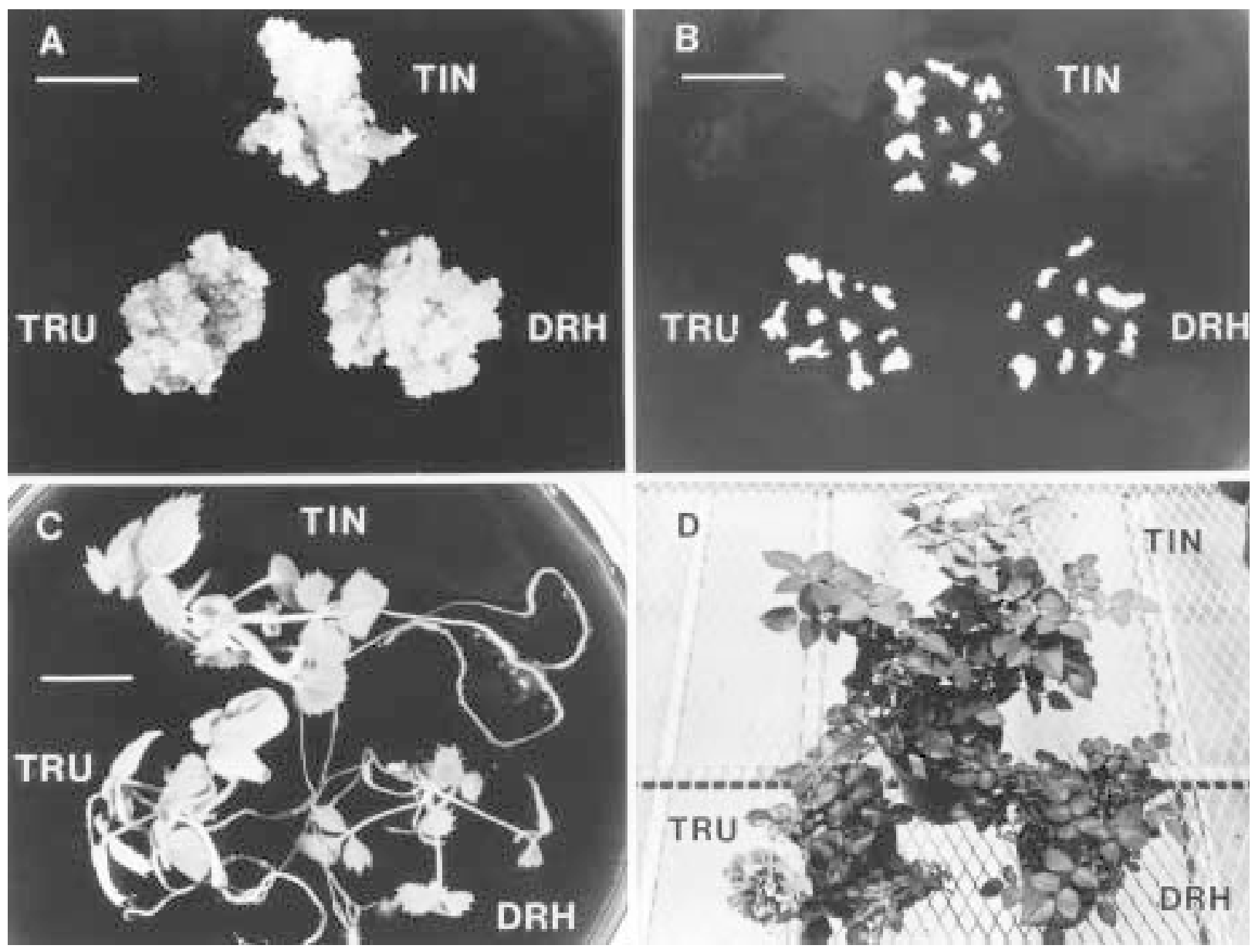

Fig. 1. (A) Embryogenic callus, (B) mature cotyledonary-stage embryos, (C) converting embryos exhibiting shoot and root development, and (D) greenhouseacclimated plants regenerated from somatic embryos of Rosa hybrida cultivars Tineké (TIN), Trumpeter (TRU), and Dr. Huey (DRH). Bars in (A), (B) and (C) represent $1 \mathrm{~cm}$. 
compared to $3 \%$ sucrose. This effect may have been partly due to increased osmotic pressure in the medium by high maltose concentration (Li et al., 1998).

There were also differences among the cultivars in their response to the four carbohydrates. 'Trumpeter' produced more mature embryos (58-59/plate) on sucrose and fructose than on glucose (35/plate) while more 'Dr. Huey' embryos (66-77/plate) matured on sucrose than on fructose (40/plate) (Fig. 2). 'Tineké' had no significant differences in the number of embryos maturing on sucrose, glucose, or fructose (35-47/plate) (Fig. 2).

Maturation of somatic embryos in response to osmotic treatments. The osmotic treatments produced varied results for the cultivars (Fig. 3). Increased osmoticum stimulated maturation of embryos only for 'Trumpeter' by addition of either $2.5 \%$ or $7.5 \%$ PEG resulting in an osmotic potential of 0.54 or $-0.62 \mathrm{MPa}$, respectively. All other osmotic treatments with myo-inositol, PEG, or mannitol either did not differ significantly from the control (3\% sucrose) or inhibited embryo maturation.

PEG did not inhibit embryo maturation with increasing concentrations possibly because the osmotic potential at the highest PEG concentration tested was lower than for all concentrations of myo-inositol and mannitol tested (Fig. 3). Another difference between the osmotica is that both myo-inositol and mannitol are metabolized by plant cells (Nelson et al., 1998; Shen et al., 1999; Wright et al., 1989) whereas PEG is a nonpermeating and non-plasmolyzing osmoticum that is not metabolized (Attree et al., 1995; Nelson et al., 1998; Shen et al., 1999; Wright et al., 1989).

Conversion of cotyledonary-stage embryos following maturation on the base medium containing various carbohydrates. The carbohydrate in the maturation medium affected conversion of 'Trumpeter' and 'Tineké' embryos, but not 'Dr. Huey'. Conversion for 'Trumpeter' was more than two times higher (27\%) when cotyledonary-stage embryos had matured on $3 \%$ fructose rather than on $3 \%$ sucrose (Fig. 4). Conversion of 'Tineké' embryos was best (12\%) after embryos had matured on $3 \%$ sucrose, $3 \%$ glucose, or $3 \%$ fructose (Fig. 4).

Conversion of embryos from 'Dr. Huey' responded quite differently from the other two cultivars (Fig. 4). Embryos of 'Dr. Huey' that matured on sucrose, glucose, or fructose did not convert, and only one of 200 embryos from the $3 \%$ maltose treatment converted.

Conversion of cotyledonary-stage embryos following maturation on the base medium containing $3 \%$ sucrose and various osmotica. All globular-stage embryos that developed to the cotyledonary-stage on maturation medium containing 3\% sucrose and an osmoticum, either myo-inositol, PEG, or mannitol, were transferred to conversion medium.

Embryos of 'Trumpeter' and 'Tineké' that matured on the maturation media with various osmotic treatments did not show any improvement in conversion rates over sucrose alone (Fig. 5).

'Dr. Huey' embryos that matured on the osmotic treatments converted and responded positively to increasing concentrations of myo-inositol and mannitol up to $5 \%$. The highest conversion rates were $39 \%$ with $2.5 \%$ mannitol and $61 \%$ with $5.0 \%$ mannitol. In comparison, conversion was significantly lower when embryos matured on myo-inositol rather than mannitol although the osmotic potential was similar. This indicated that the rate of conversion was affected by the

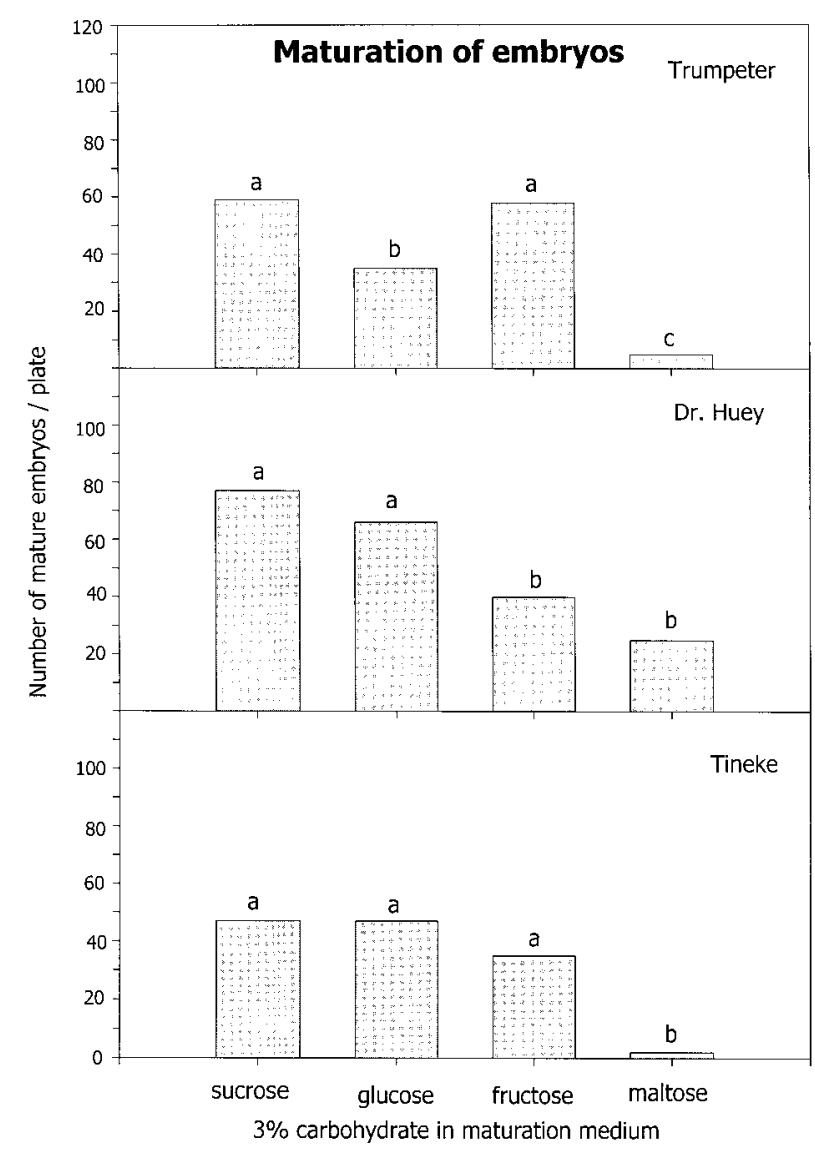

Fig. 2. Mean number of cotyledonary-stage embryos per plate 4-5 weeks after $2 \mathrm{~mL}$ of cell suspension containing globular-stage embryos of three Rosa hybrida cvs. ('Trumpeter', 'Tineké', and 'Dr. Huey') had been placed on one of four maturation media differing by carbohydrate source $(3 \%$ sucrose, glucose, fructose, or maltose). Means (three replicates of 50 embryos each) followed by different letters are significantly different at $P \leq 0.05$ according to Duncan's new multiple range test done separately for each cultivar.

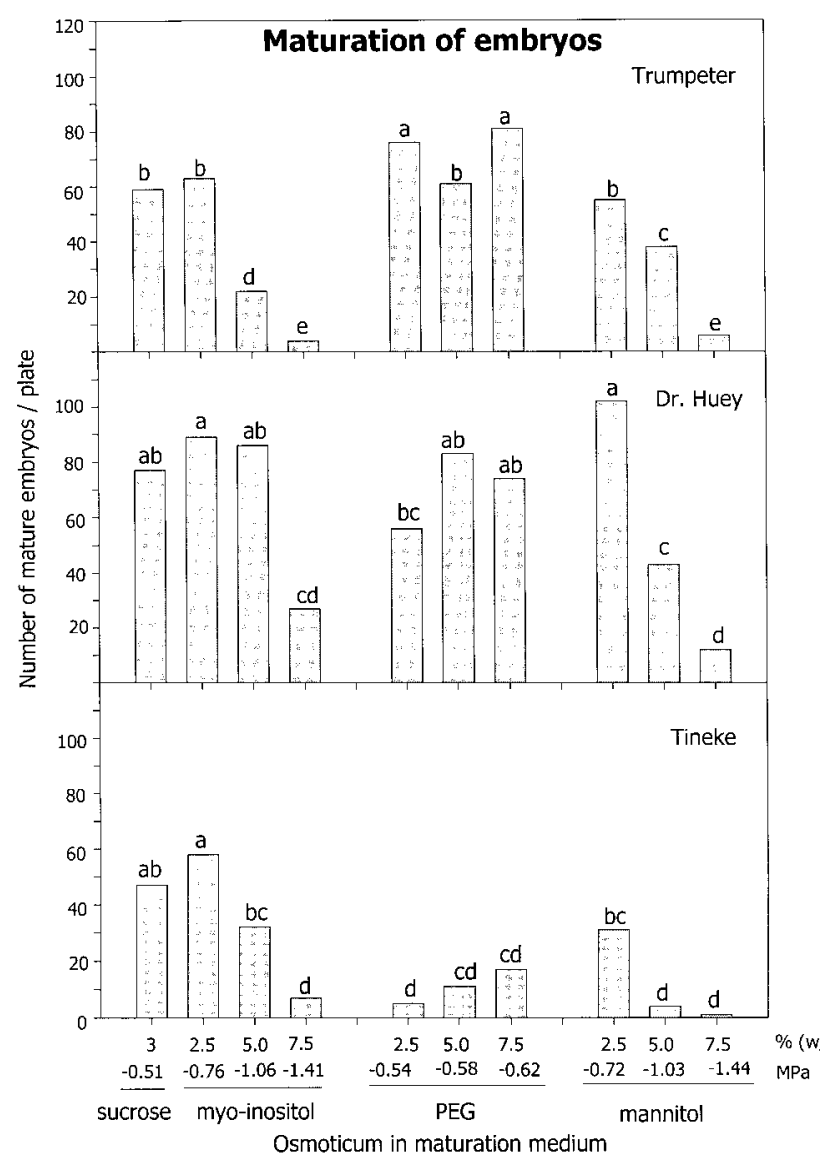

Fig. 3. Mean number of cotyledonary-stage embryos per plate 4-5 weeks after 2 $\mathrm{mL}$-cell suspension containing globular stage embryos of three Rosa hybrida cvs. ('Trumpeter', 'Tineké', and 'Dr. Huey') had been placed on one of nine maturation media containing $3 \%$ sucrose and supplemented with an osmoticum (myo-inositol, PEG, or mannitol) at different concentrations as shown on the $\mathrm{X}$ axis as percentage of (w/v) and osmotic potential (MPa). Means (three replicates of 50 embryos each) followed by different letters are significantly different at $P \leq 0.05$ according to Duncan's new multiple range test done separately for each cultivar. 


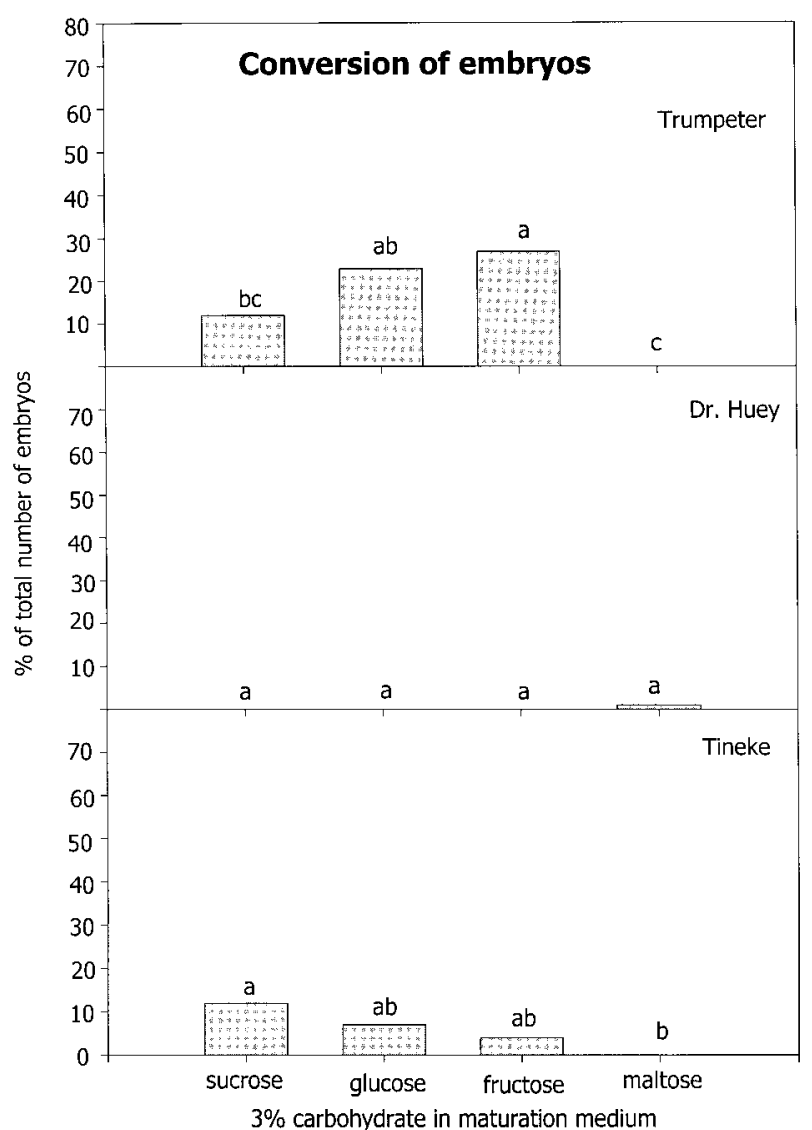

Fig. 4. Percentage of cotyledonary-stage embryos of three Rosa hybrida cvs. ('Trumpeter', 'Dr. Huey', and 'Tineké') by carbohydrate source (3\% sucrose, glucose, fructose, or maltose) as shown on the $\mathrm{X}$ axis. Means (three replicates of 50 embryos each) followed by different letters are significantly different at $P \leq 0.05$ according to Duncan's new multiple range test done separately for each cultivar.

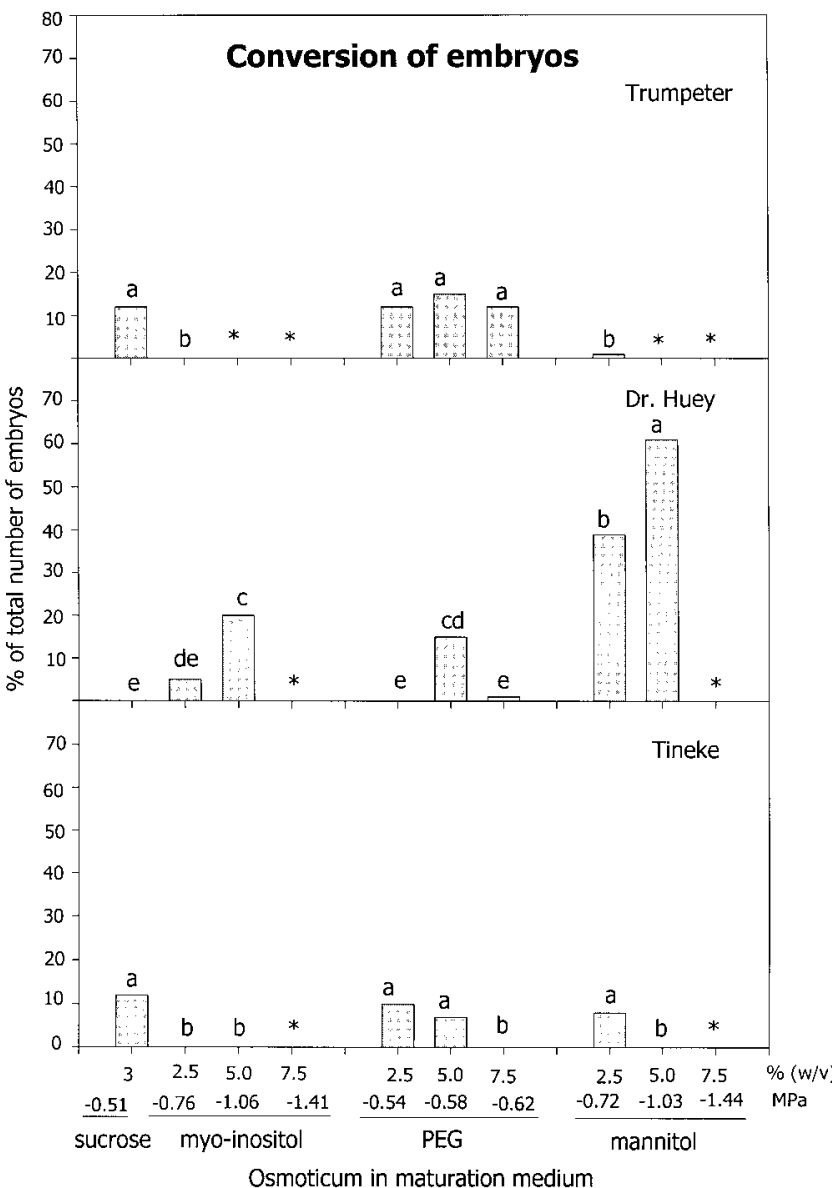

Fig. 5. Percentage of cotyledonary-stage embryos of three Rosa hybrida cvs. ('Trumpeter', 'Dr. Huey', and 'Tineké') that converted to form roots and shoots 6 weeks after the cotyledonary-stage embryos had been placed on conversion medium (MS salts and 3\% sucrose). These embryos had matured to the cotyledonary stage on one of nine maturation media containing 3\% sucrose and supplemented with an osmoticum (myoinositol, PEG, or mannitol). The osmoticum in the maturation media are shown on the $\mathrm{X}$ axis as percentage of (w/v) and osmotic potential (MPa). Means (three replicates of 50 embryos each) followed by different letters are significantly different at $P \leq 0.05$ according to Duncan's new multiple range test done separately for each cultivar. ${ }^{*}$ No data collected due to insufficient embryo maturation. osmoticum and not the osmotic potential resulting from the addition of the osmoticum.

All cultivars had normal shoot and root development (Fig. 1 C and D).

\section{Conclusions}

High rates of somatic embryo maturation and conversion of multiple rose cultivars is required for development of efficient transformation systems of this horticulturally important crop. This study demonstrated that various carbohydrates or osmoticum could be used to improve maturation, and more importantly, conversion of somatic embryos from rose cultivars. Conversion above $60 \%$ was obtained for 'Dr. Huey' following maturation on 5\% mannitol, $27 \%$ for 'Trumpeter' on 3\% fructose, and $12 \%$ for 'Tineké' embryos on $3 \%$ sucrose. This study also demonstrated that treatment effects are highly cultivarspecific for roses and validates the need to optimize regeneration protocols for individual cultivars.

\section{Literature Cited}

Attree, S.M., M.K. Pomeroy, and L.C. Fowke. 1995 Development of white spruce (Picea glauca (Moench.) Voss) somatic embryos during culture with abscisic acid and osmoticum, and their tolerance to drying and frozen storage. J. Expt. Bot. 46:433-439.

Biahoua, A. and L. Bonneau. 1999. Control of in vitro somatic embryogenesis of the spindle tree (Euonymus europaeus L.) by the sugar type and the osmotic potential of the culture medium. Plant Cell Rpt. 19:185-190.

Derks, F.H.M., A.J. van Dijk, C.H. Hänisch ten Cate, D.E.A. Florack, L.A.M. Dubois, and D.P. de Vries. 1995. Prolongation of vase life of cut roses via introduction of genes coding for antibacterial activity, somatic embryogenesis and Agrobacterium-mediated transformation. Acta Hort. 405:205-209.

de Wit, J.C., H.F. Esendam, J.J. Honkanen, and U. Tuominen. 1990. Somatic embryogenesis and regeneration of flowering plants of rose. Plant Cell Rpt. 9:456-458.

Firoozabady, E., Y. Moy, N. Courtney-Gutterson, and K. Robinson. 1994. Regeneration of transgenic rose (Rosa hybrida) plants from em- bryogenic tissue. Bio/Technology 12:609-613.

Hsia, C. and S.S. Korban. 1996. Organogenesis and somatic embryogenesis in callus cultures of Rosa hybrida and Rosa chinensis minima. Plant Cell Tiss. Org. Cult. 44:1-6.

Kintzios, S., C. Manos, and O. Makri. 1999. Somatic embryogenesis from mature leaves of rose (Rosa sp.). Plant Cell Rpt. 18:467-472.

Klimaszewska, K., Y. Devantier, D. Lachance, M.A Lelu, and P.J. Charest. 1997. Larix laricina. (tamarack): Somatic embryogenesis and genetic transformation. Can. J. For. Res. 27:538-550.

Kunitake, H., H. Imamizo, and M. Mii. 1993. Somatic embryogenesis and plant regeneration from immature seed-derived calli of rugosa rose (Rosa rugosa Thunb.). Plant Sci. 90:187-184.

Li, X.Y., F.H. Huang, J.B. Murphy, and E.E. Gbur, Jr. 1998. Polyethylene glycol and maltose enhance somatic embryo maturation in loblolly pine (Pinus taeda L.). In Vitro Cell. Dev. Biol.Plant 34:22-26.

Marchant, R., M.R. Davey, J.A. Lucas, and J.B. Power. 1996. Somatic embryogenesis and plan regeneration in Floribunda rose (Rosa hybrida L.) cvs. Trumpeter and Glad Tidings. Plant Sci. 120:95-105.

Marchant, R., J.B. Power, J.A. Lucas, and M.R. 
Davey. 1998. Biolistic transformation of rose (Rosa hybrida L.). Ann. Bot. 81:109-114.

Matthews, D., J. Mottley, I. Horan, and A.V. Roberts. 1991. A protoplast to plant system in roses. Plant Cell Tiss. Org. Cult. 24:173-180.

Murali. S., D. Sreedhar, and T.S. Lokeswari. 1996 Regeneration through somatic embryogenesis from petal-derived calli of Rosa hybrida $\mathrm{L}$. cv Arizona (hybrid tea). Euphytica 91:271-275.

Murashige, T. andF. Skoog. 1962. A revised medium for rapid growth and bioassays with tobacco tissue cultures. Physiol. Plant. 15:473-497.

Nelson, D.E., G. Rammesmayer, and H.J. Bohnert. 1998. Regulation of cell-specific inositol metabolism and transport in plant salinity tolerance. Plant Cell 10:753-764.

Noriega, C. and M.R. Sondahl. 1991. Somatic embryogenesis in hybrid tea roses. Bio/Technology 9:991-993.

Sarasan, V., A.V. Roberts, and G.R. Rout. 2001. Methyl laurate and 6-benzyladenine promote the germination of somatic embryos of a hybrid rose. Plant Cell Rpt. 20:183-186.

Schenk, R.U. and A.C. Hildebrandt. 1972. Medium and techniques for induction and growth of monocotyledonous and dicotyledonous plant cell cultures. Can. J. Bot. 50:199-204.

Schuller, A. and G. Reuther. 1993. Response of Abies alba embryonal-suspensor mass to various carbohydrate treatments. Plant Cell Rpt 12:199-202.

Shen, B., S. Hohmann, R.G. Jensen, and H.J. Bohnert. 1999. Roles of sugar alcohols in osmotic stress adaptation. Replacement of glycerol by mannitol and sorbitol in yeast. Plant Physiol. 121:45-52.

Souq, F., P. Coutos-Thevenot, H. Yean, G. Delbard, Y. Maziere, J.P. Barbem, and M. Boulay. 1996. Genetic transformation of roses, 2 examples: One on morphogenesis, the other on anthocyanin biosynthetic pathway. Acta Hort. 424:381-388.

Tremblay, L. and F.M. Tremblay. 1991. Carbohy- drate requirements for the development of black spruce (Picea mariana Mill BSP) and red spruce (Picea rubens Sarg) somatic embryos. Plant Cell Tiss. Org. Cult. 27:95-103.

Valles, J. and P. Boxus. 1987. Regeneration from Rosa callus. Acta Hort. 211:691-696.

van der Salm, T.P.M., C.J.G. van der Toorn, R. Bouwer, C.H. Hänisch ten Catem, and H.J.M. Dons. 1996. Somatic embryogenesis and shoot regeneration from excised adventitious roots of the rootstock Rosa hybrida L. 'Moneyway'. Plant Cell Rpt. 15:522-526.

van der Salm, T.P.M., C.J.G. van der Toorn, R. Bouwer, C.H. Hänisch ten Catem, and H.J.M. Dons. 1997. Production of ROL gene transformed plants of Rosa hybrida $\mathrm{L}$ and characterization of their rooting ability. Mol. Breeding 3:39-47.

Wright, P.J., R. Fosterm, and R.H. Reed. 1989. Turnover of the intracellular mannitol pool of Fucus spiralis L. (Fucales, Phaeophyta) during osmotic shock. J. Expt. Bot. 40:1347-1353. 